\title{
Recommendations for Specimen and Therapy Selection in Colorectal Cancer
}

Snehal B. Patel · Robert Bookstein - Navid Farahani - Myriam Chevarie-Davis ·

Andy Pao · Angela Aguiluz · Christian Riley · Jennelle C. Hodge •

Serhan Alkan · Zhenqui Liu · Nan Deng · Jean R. Lopategui

Received: January 20, 2021 / Accepted: March 31, 2021 / Published online: April 25, 2021

(C) The Author(s) 2021

\begin{abstract}
Introduction: Next-generation sequencing has emerged as a clinical tool for the identification of actionable mutations to triage advanced colorectal cancer patients for targeted therapies. The literature is conflicted as to whether primaries or their metastases should be selected for sequencing. Some authors suggest that either site may be sequenced, whereas others recommend sequencing the primary, the metastasis, or even both tumors. Here, we address this issue head on with a meta-analysis and provide for the first time a set of sensible recommendations to make this determination.
\end{abstract}

Supplementary Information The online version contains supplementary material available at https:// doi.org/10.1007/s40487-021-00151-7.

S. B. Patel · R. Bookstein · N. Farahani .

M. Chevarie-Davis - A. Pao - A. Aguiluz - C. Riley ·

J. C. Hodge · S. Alkan · J. R. Lopategui $(\bowtie)$

Cedars-Sinai Medical Center, Division of Molecular Pathology and Cytogenetics, Department of Pathology and Laboratory Medicine, 8700 Beverly Blvd., SSB \#362, Los Angeles, CA 90048, USA e-mail: Jean.Lopategui@cshs.org

S. B. Patel

HeloGenika LLC, Dexter, MI 48130, USA

Z. Liu $\cdot$ N. Deng

Cedars-Sinai Medical Center, Biostatistics and Bioinformatics Research Center, Samuel Oschin Comprehensive Cancer Institute, Los Angeles, CA, USA
Methods: From our own series, we include 43 tumors from 13 patients including 14 primaries, 10 regional lymph node metastases, 17 distant metastases, and two anastomotic recurrences sequenced using the 50 gene Ion AmpliSeq cancer NGS panel v2.

Results: Based on our new cohort and a metaanalysis, we found that $\sim 77 \%$ of patient-matched primary-metastatic pairs have identical alterations in these 50 cancer-associated genes. Conclusions: Low tumor cellularity, tumor heterogeneity, clonal evolution, treatment status, sample quality, and/or size of the sequencing panel accounted for a proportion of the differential detection of mutations at primary and metastatic sites. The therapeutic implications of the most frequently discordant alterations (TP53, APC, PIK3CA, and SMAD4) are discussed. Our meta-analysis indicates that a subset of patients who fail initial therapy may benefit from sequencing of additional sites to identify new actionable genomic abnormalities not present in the initial analysis. Evidencebased recommendations are proposed.

Keywords: Colorectal cancer; Next-generation sequencing; Paired primary and metastatic tumors; Patient-matched tumors; Specimen selection; Therapy selection; Tumor heterogeneity 


\section{Key Summary Points}

Why carry out this study?

Next-generation sequencing (NGS) is becoming the modality of choice for informing therapeutic decisions for patients with advanced-stage disease.

Previous studies sequencing primary and metastatic colorectal cancers have found differing levels of mutational concordance and have reached different conclusions regarding whether primaries or their metastases should be analyzed.

The study asked "what is the preferred sample for NGS, the primary or metastatic site?" Oncologists and pathologists need evidence-based guidelines to help with specimen selection for sequencing, as the decision may have significant impact on therapy, clinical trial enrollment, and possibly patient outcomes.

\section{What was learned from the study?}

Our data and meta-analysis show high mutational concordance for actionable mutations between primary and metastatic sites. However, over $20 \%$ are discordant and may identify new actionable mutations in resistant patients.

We present evidence-based recommendations that combine clinical considerations and pathologic parameters to help oncologists and pathologists arrive at a sensible approach to specimen selection.

\section{DIGITAL FEATURES}

This article is published with digital features, including a summary slide, to facilitate understanding of the article. To view digital features for this article, go to https://doi.org/10.6084/ m9.figshare.14340374.

\section{INTRODUCTION}

Colorectal carcinoma (CRC) is the third leading cause of cancer and cancer mortality in both men and women in the United States with 149,500 new cases and 52,980 deaths projected in 2021 [1]. Approximately $20-25 \%$ of CRC patients have metastatic disease (mCRC) at the time of diagnosis, and the majority of these are incurable [2]. The treatment for mCRC may be resection of the primary CRC with or without metastasectomy followed by adjuvant chemotherapy [3, 4]; however, standard chemotherapy has limited benefit in prolonging survival.

Over the past decade, our improved understanding of CRC biology has resulted in the identification of novel molecular targets with corresponding targeted therapies which improve survival and have fewer side effects [5]. The most important of these is the protooncogene epidermal growth factor receptor (EGFR), which is often overexpressed in CRC $[6,7]$. Two monoclonal antibodies, cetuximab and panitumumab, which target the extracellular ligand-binding domain of EGFR, are FDAapproved for treating mCRC lacking KRAS- and NRAS-activating mutations [8]. CRCs that harbor RAS-activating mutations, however, are resistant to anti-EGFR antibodies, and these therapies are contraindicated in this setting. Similarly, CRCs that harbor BRAF-activating mutations are typically resistant to anti-EGFR antibodies; however, combining anti-EGFR therapy with the BRAF V600E inhibitor encorafenib has demonstrated efficacy [9]. Given the therapeutic relevance of $K R A S, N R A S$, and $B R A F$ mutations, testing for all three is recommended in the metastatic setting $[3,4,10]$. Many have found mutations in these genes to be highly concordant in paired primary and metastatic 
colorectal cancers [11], leading to the NCCN guideline that either tumor is suitable for genotyping these markers [3, 4]. Conversely, others have found significant discordance in KRAS status between sites and after therapy [12-15].

Looking beyond RAS and BRAF mutations, about $15 \%$ of CRCs harbor DNA mismatch repair deficiency (dMMR), which results in microsatellite instability (MSI) [16]. The identification of this subset of CRCs is critical due to its prognostic and therapeutic implications [16]. First, patients with dMMR/MSI tumors tend to have significantly more favorable survival outcomes than those with MMR-proficient/microsatellite stable tumors. Second, dMMR/MSI tumors have poor response to standard CRC therapy with FOLFOX/FOLFIRI regimens but are exquisitely sensitive to immunotherapies targeting immune checkpoint molecules. Indeed, several immunotherapies are now FDA-approved for treating dMMR/MSI CRC [17]. Several studies have demonstrated a high degree of concordance in dMMR/MSI status between patient-matched primary and metastatic CRCs, but site-specific and treatment-induced differences were noted [18-22].

More recently, targeted next-generation sequencing (NGS) has entered the clinical arena as a cost-effective means of identifying additional actionable genetic abnormalities [23, 24]. Thus, for those CRC patients who are refractory to cytotoxic chemotherapy or are not candidates for anti-EGFR antibody therapy or checkpoint inhibitors, NGS may identify additional therapeutic targets beyond RAS, BRAF, and MSI and allow for placement in clinical trials. This raises the question of whether paired primary and metastatic CRCs will be concordant for these additional targets.

One reason to expect otherwise is that tumors evolve over time and after branching, continue to evolve over time independently. Therefore, genetic differences within different areas of a tumor mass (intratumoral heterogeneity) and between paired primary and metastatic tumors (intertumoral heterogeneity) are to be expected. While this phenomenon of genetic heterogeneity of tumors is generally accepted, the qualitative and quantitative extent of the heterogeneity between primary tumors (PTs) and their paired metastases to either distant organs (DMs) or regional lymph nodes (RLNs) is controversial. Metastases may potentially harbor a distinct spectrum of mutations compared to their PTs and therefore, may respond to a different set of drugs. Genetic discordance creates a challenge for the pathologist who must determine which formalin-fixed paraffin-embedded (FFPE) tissue block to select for NGS, a decision which may profoundly impact downstream therapeutics and ultimately patient outcomes.

Several groups have attempted to address this problem using NGS but have had conflicting results with some finding a high degree of concordance [25-28] and others finding a high degree of discordance [29]. Likewise, these groups have come to greatly differing conclusions regarding whether the decision of specimen selection is relevant at all and, if so, whether one or both sites should be analyzed. Herein, we compare the mutational spectrum of primary and metastatic CRCs using NGS with a 50 cancer-associated gene panel, present the first meta-analysis on the topic of mutational concordance in CRCs, and suggest some general guidelines to help with this dilemma of great clinical importance.

\section{METHODS}

\section{Patient and Specimen Selection}

Approval with a waiver for consent was obtained from the Cedars-Sinai Medical Center (CSMC) Institutional Review Board. To evaluate mutational concordance between PTs and metastases, CSMC pathology results between 2013 and 2014 were searched for patients with confirmed CRCs based on clinical, morphologic, and/or immunohistochemical features and who had archival tissue from both a PT and DM. Patients who had a history of a second cancer type were excluded to avoid confusion when assessing mutational concordance.

Hematoxylin and eosin (H\&E)-stained slides were reviewed by two pathologists (J.R.L plus M.C.D., N.F., or S.B.P) to select the optimal FFPE 
blocks from the PT and DM, as well as RLN and anastomotic recurrences when present, for NGS. Tumors with scant tissue or extensive necrosis were excluded. Tumors with $<20 \%$ tumor content (TC) were sequenced but excluded from pairwise mutational concordance analysis of the patient-matched primary tumor and its metastasis, as low TC is known to yield false negatives. When multiple sections of the tumor were available, the section with the greatest viable tumor cellularity was selected. When indicated, viable tumor on H\&E-stained slides was outlined to enrich for TC. Two to four $10-\mu \mathrm{m}$-thick unstained tissue sections were prepared on uncharged slides.

\section{DNA Extraction}

The entire section or, when applicable, the outlined area was scraped from the unstained slides. Genomic DNA was extracted from the tissue using the QIAcube (9001882; Qiagen, Hilden, Germany) according to manufacturer's protocol. The purified DNA was subsequently quantitated using the Qubit 2.0 Fluorometer
(Q32866; Thermo Fisher Scientific, Carlsbad, CA).

\section{Next-Generation Sequencing}

NGS was performed on the Ion Torrent Personal Genome Machine (4462921; Thermo Fisher Scientific) using the Ion AmpliSeq Cancer Hotspot Panel version 2 (4475346; Thermo Fisher Scientific) according to manufacturer's protocol. The 50 cancer-associated genes evaluated by this panel are listed in Table 1 . The specific regions of the genes interrogated by the panel are available from the manufacturer. Briefly, a library of 207 amplicons was generated in a multiplex PCR using $10 \mathrm{ng}$ of purified genomic DNA. Unique Ion Xpress barcodes (4474517; Thermo Fisher Scientific) were ligated to each sample. Barcoded libraries were purified, equalized, and combined for templating on ion sphere particles (4474517; Thermo Fisher Scientific). Templated ion sphere particles were enriched, loaded onto the 314 chip, and sequenced on the Personal Genome Machine.

Table 1 Fifty genes interrogated by the AmpliSeq Cancer Hotspot Panel v2

\begin{tabular}{lllllll}
\hline ABL1 & AKT1 & ALK & APC & ATM & BRAF & CDH1 \\
CDKN2A & CSF1R & CTNNB1 & EGFR & ERBB2 & ERBB4 & EZH2 \\
FBXW7 & FGFR1 & FGFR2 & FGFR3 & FLT3 & GNA11 & GNAQ \\
GNAS & HNF1A & HRAS & IDH1 & IDH2 & JAK2 & JAK3 \\
KDR & KIT & KRAS & MET & MLH1 & MPL & NOTCH1 \\
NPM1 & NRAS & PDGFRA & PIK3CA & PTEN & PTPN11 & RB1 \\
RET & SMAD4 & SMARCB1 & SMO & SRC & STK11 & TP53 \\
VHL & & & & & \\
\hline
\end{tabular}

The panel includes tumor suppressor genes and oncogenes recurrently mutated in cancer. In bold are the recurrently mutated genes in colorectal cancer. APC (81\%), TP53 (60\%), KRAS (43\%), PIK3CA (18\%), FBXW7 (11\%), SMAD4 (10\%), NRAS (9\%), and CTNNB1 (5\%) were the most highly mutated genes in non-hypermutated colorectal cancers, whereas APC (51\%), BRAF (46\%), FBXW7 (46\%) ATM (40\%), PIK3CA (34\%), KRAS (31\%), and MLH1 (14\%) were among the most commonly mutated genes in hypermutated colorectal cancers (The Cancer Genome Atlas database [30]). 


\section{Bioinformatics Pipeline}

Signal processing, base calling, and alignment were performed using Torrent Suite software version 2.0 (Thermo Fisher Scientific, Waltham, MA, USA). To pass quality control requirements, samples must have $>250 \mathrm{X}$ coverage for at least 203 of the 207 amplicons on the panel. Variant calling was performed using NextGENe version 2.3.3 (SoftGenetics, State College, PA, USA) and Torrent Suite version 2.0. Manual review of alignments was performed using the NextGENe version 2.3.3 viewer (SoftGenetics). Only nonsynonymous variants with quality scores $>25$, coverage $>250$ reads, similar forward/reverse ratios, and variant allele frequencies (VAFs) $>$ $5 \%$ were evaluated. Calls in homopolymer regions longer than 3nt were excluded. Polymorphic variants identified in the 1000 Genomes database were excluded if the variant was present in $>10 \%$ of the population, which makes it highly unlikely to be pathogenic, or if literature supported it being benign.

\section{Meta-Analysis}

PubMed was interrogated with the following search criteria: (colon OR rectal OR colorectal) AND (cancer OR neoplasm OR tumor) AND (primary AND metastatic) AND ("next generation sequencing" OR "next-generation sequencing" OR "massively parallel sequencing" OR "targeted sequencing" OR "exome sequencing" OR "genome sequencing" OR "molecular profiling") AND (comparison OR paired OR pairwise OR "patient-matched" OR matched OR "primary-metastatic" OR concordance OR discordance). Search results were individually reviewed. Studies with insufficient methods for teasing out CRCs from other tumors or insufficient NGS panel information were excluded. Five studies that compared results of NGS in primary and metastatic CRC were included. Factors that potentially influence concordance rates, including number of genes on the panel, tumor purity cutoffs, mean coverage, and VAF cutoffs, were also extracted. The total number of primary-metastatic pairs and concordant pairs were extracted from each publication. The
Table 2 Clinical and specimen summary

\begin{tabular}{|c|c|}
\hline Clinical & \\
\hline Patients, $n$ & 13 \\
\hline Age, mean \pm SD (years) & $60 \pm 18$ \\
\hline Sex, male/female & $8: 5$ \\
\hline \multicolumn{2}{|c|}{ Pathologic stage at diagnosis, $n$} \\
\hline I & 1 \\
\hline II & 3 \\
\hline III & 1 \\
\hline IV & 8 \\
\hline \multicolumn{2}{|l|}{ Histologic differentiation, $n$} \\
\hline Well-moderate & 2 \\
\hline Moderate & 6 \\
\hline Moderate-poor & 3 \\
\hline Treated, N/A & 2 \\
\hline \multicolumn{2}{|l|}{ Chemo/radiotherapy, $n$} \\
\hline Neoadjuvant & 3 \\
\hline Adjuvant & $10 / 10^{*}$ \\
\hline \multicolumn{2}{|l|}{ Specimens } \\
\hline \multicolumn{2}{|l|}{ Primary site, $n$} \\
\hline Cecum & 1 \\
\hline Ascending colon & 2 \\
\hline Transverse colon & 2 \\
\hline Descending colon & 1 \\
\hline Sigmoid colon & 2 \\
\hline Rectosigmoid & 3 \\
\hline Rectum & 3 \\
\hline Total & $14^{\#}$ \\
\hline \multicolumn{2}{|l|}{ Distant metastatic site, $n$} \\
\hline Liver & 8 \\
\hline Lung & 2 \\
\hline Distant lymph node & 3 \\
\hline Peritoneum & 2 \\
\hline Small bowel & 1 \\
\hline Presacral soft tissue & 1 \\
\hline
\end{tabular}


Table 2 continued

\begin{tabular}{ll}
\hline Clinical & \\
\hline Total & 17 \\
Regional lymph node metastases, $n$ & 10 \\
Anastomotic recurrences, $n$ & 2 \\
Surgery & 3 \\
Biopsy & 31 \\
Resection & \\
\hline
\end{tabular}

*Three patients could not be verified as having received adjuvant therapy, so denominators were adjusted accordingly

\# One patient had two primaries number of concordant pairs were redetermined after considering only the 50 genes on the Ion AmpliSeq Cancer Hotspot Panel version 2 to allow for a fair comparison across all studies.

\section{Statistical Analysis}

The two-tailed Fisher's exact test with $\alpha=0.05$ was used to compare proportions. For multiple pairwise comparisons, a Bonferroni correction was applied to control the type I error rate. Statistical analyses were performed by S.B.P., N.D, and Z.L. The meta (Version 4.15-1) and metafor (Version 2.4-0) packages for $\mathrm{R}$ programming language (Version 4.0.2) were used for the meta-analysis and for generating diagnostic plots and forest plots. To identify outlier studies, leave-one-out analysis and Baujat plots were used to evaluate for disproportionate influence of any given study. No

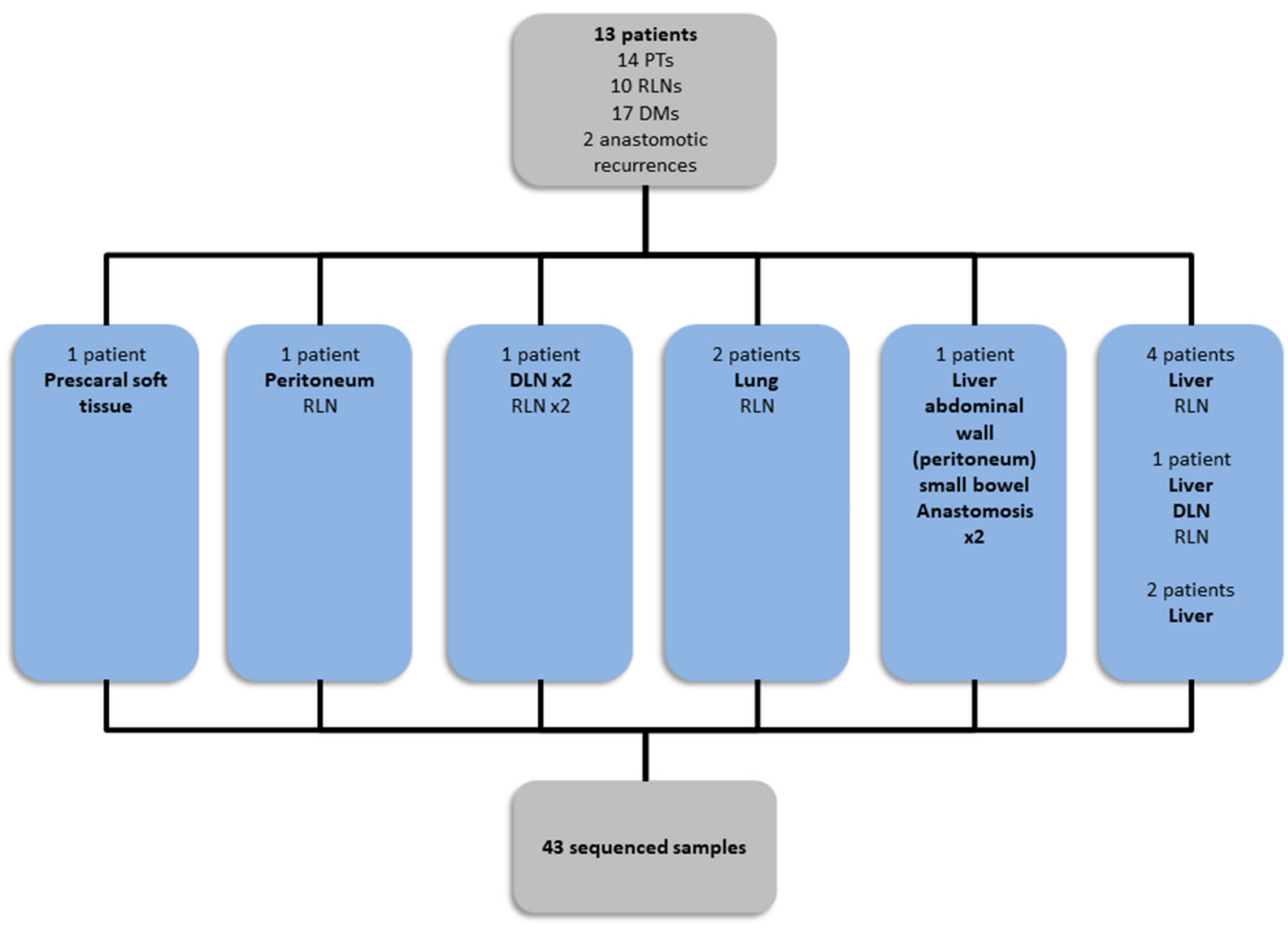

Fig. 1 Distribution of samples. PTs primary tumors, RLNs regional lymph node metastases, DLNs distant lymph node metastases, $D M s$ distant organ metastases. Note that one patient had two synchronous primaries 
transformations were applied to the data, a random effects model was selected for calculating effect sizes, and the DerSimonian-Laird method was used to estimate $\tau$.

\section{RESULTS}

\section{Clinical and Pathologic Characteristics}

To compare mutational differences between primary and metastatic CRCs, 13 patients with available clinical data and paired tumors were included in the study (Table 2, Supplemental Table S1). Eight had pathologic stage IV disease at diagnosis (synchronous), and five developed metastases subsequently (metachronous). The time interval from colectomy to first metastasectomy averaged 1.7 years (0.5-4.8 years). Forty-three tumors were sequenced, including 14 PTs, 10 RLNs, 17 DMs, and two anastomotic recurrences (Fig. 1, Table 2). One patient had two synchronous PTs, three had no RLNs (pNO), and one had only a microscopic RLN, which was not suitable for NGS.

None had clinical evidence of other malignancies, colorectal polyposis, or Lynch syndrome/hereditary non-polyposis colorectal cancer. Two patients had PTs with immunohistochemical evidence of loss of mismatch repair protein expression (Supplemental Table S1). Both tumors were right-sided and had morphologic features of microsatellite instable tumors. One had a BRAF V600E mutation (patient 10), which effectively excludes Lynch syndrome. While the other (patient 6) did not have a BRAF mutation, only about half of sporadic microsatellite instable tumors harbor these [23].

Two tumors (PT in patient 8 and liver DM in patient 4) had TC less than $20 \%$, our clinical laboratory-established threshold for adequacy. While these samples were sequenced for completeness, we excluded them from concordance calculations.
Table 3 Mutation rates and discordance rates in primarymetastatic tumor pairs

\begin{tabular}{|c|c|c|}
\hline & $\begin{array}{l}\text { PT: DM } \\
\left(n=12 \text { pairs }^{\#}\right)\end{array}$ & $\begin{array}{l}\text { PT: RLN } \\
(n=9 \text { pairs })\end{array}$ \\
\hline $\begin{array}{l}\text { Total mutations*, } n: \\
n\end{array}$ & 21: 17 & 16: 14 \\
\hline $\begin{array}{l}\text { Mutation rate, } \\
\text { mutations/tumor }\end{array}$ & 1.8: 1.4 & 1.8: 1.6 \\
\hline \multicolumn{3}{|l|}{ Mutations } \\
\hline Concordant, $n$ & $17(83 \%)$ & $14(82 \%)$ \\
\hline Discordant, $n$ & $4(17 \%)$ & $3(18 \%)$ \\
\hline \multicolumn{3}{|l|}{ Tumor pairs } \\
\hline Concordant, $n$ & $10(83 \%)$ & $7(80 \%)$ \\
\hline Overlapping, $n$ & $1(8 \%)$ & $1(10 \%)$ \\
\hline Discordant, $n$ & $1(8 \%)$ & $1(10 \%)$ \\
\hline $\begin{array}{l}\text { Number of } \\
\text { mutations, } n: n: n\end{array}$ & $\begin{array}{l}\text { Both: PT only: } \\
\text { DM only }\end{array}$ & $\begin{array}{l}\text { Both: PT only: } \\
\text { RLN only }\end{array}$ \\
\hline$K R A S$ & 2: $0: 0$ & 2: $0: 0$ \\
\hline$N R A S$ & $0: 1: 0$ & - \\
\hline$B R A F$ & 1: 0: 0 & 1: 0: 0 \\
\hline TP53 & 7: 1: 0 & 5: 1: 0 \\
\hline$A P C$ & 3: 1: 0 & 3: $0: 0$ \\
\hline PIK3CA & 1: 1: 0 & 1: 1: 1 \\
\hline$A T M$ & 1: 0: 0 & 1: 0: 0 \\
\hline PTEN & 1: 0: 0 & 1: $0: 0$ \\
\hline$F B X W 7$ & 1: $0: 0$ & 1: $0: 0$ \\
\hline
\end{tabular}

PTs primary tumors, DMs distant metastases, RLNs regional lymph node metastases

\# For patients 4 and 8 , the liver metastasis and PT, respectively, were excluded from analysis. In a clinical setting, these tumors which had inadequate tumor cellularity $(<20 \%)$ would not be sequenced when another sample with adequate tumor cellularity was concurrently available

*To avoid falsely inflated contribution from patients with multiple metastases, they were counted as a single metastasis 




Fig. 2 Prevalence of mutations across primary and metastatic tumors. PTs primary tumors, RLNs = regional lymph node metastases, $D L N s$ distant lymph node metastases, $D M s$ distant organ metastases

\section{Gross Comparison of Mutational Burden and Spectra of Primaries and Metastases}

To obtain a broad view of whether there are dramatic differences between primary-metastatic pairs, we compared the overall and genespecific mutational burden in PTs, RLNs, and DMs. Interestingly, metastatic tumors had mutational burdens that were generally similar to PTs (Table 3), and the spectrum of genes mutated and their prevalence were also comparable (Fig. 2). In descending order, TP53, APC, and KRAS were the three most frequently mutated genes, which is somewhat different from the relative frequencies in the Cancer Genome Atlas database [30], possibly related to more advanced disease in our cohort (Table 1).

\section{Pairwise Genetic Comparison of Primaries and Metastases}

Next, we address the crux of this study, the extent to which metastases differ genetically from the primary from which they originated. In our cohort, metastases generally had similar mutations as their PTs (Tables 3, 4). Patient 8 was excluded from pairwise comparison between PT and DM due to low TC (15\%) of its PT. The liver DM from patient 4 had a low TC $(10 \%)$ and therefore, this site was excluded from analysis. However, the patient was included in the concordance analysis, as he had multiple other DMs with adequate TC. Mutational spectra in PT-DM pairs were identical in 10 of 12 (83\%) patients but only partially overlapping in patient $5(8 \%)$ and entirely discordant in patient $6(8 \%)$. Among the PT-DM pairs, a total of 21 mutations were detected, $17(83 \%)$ of 







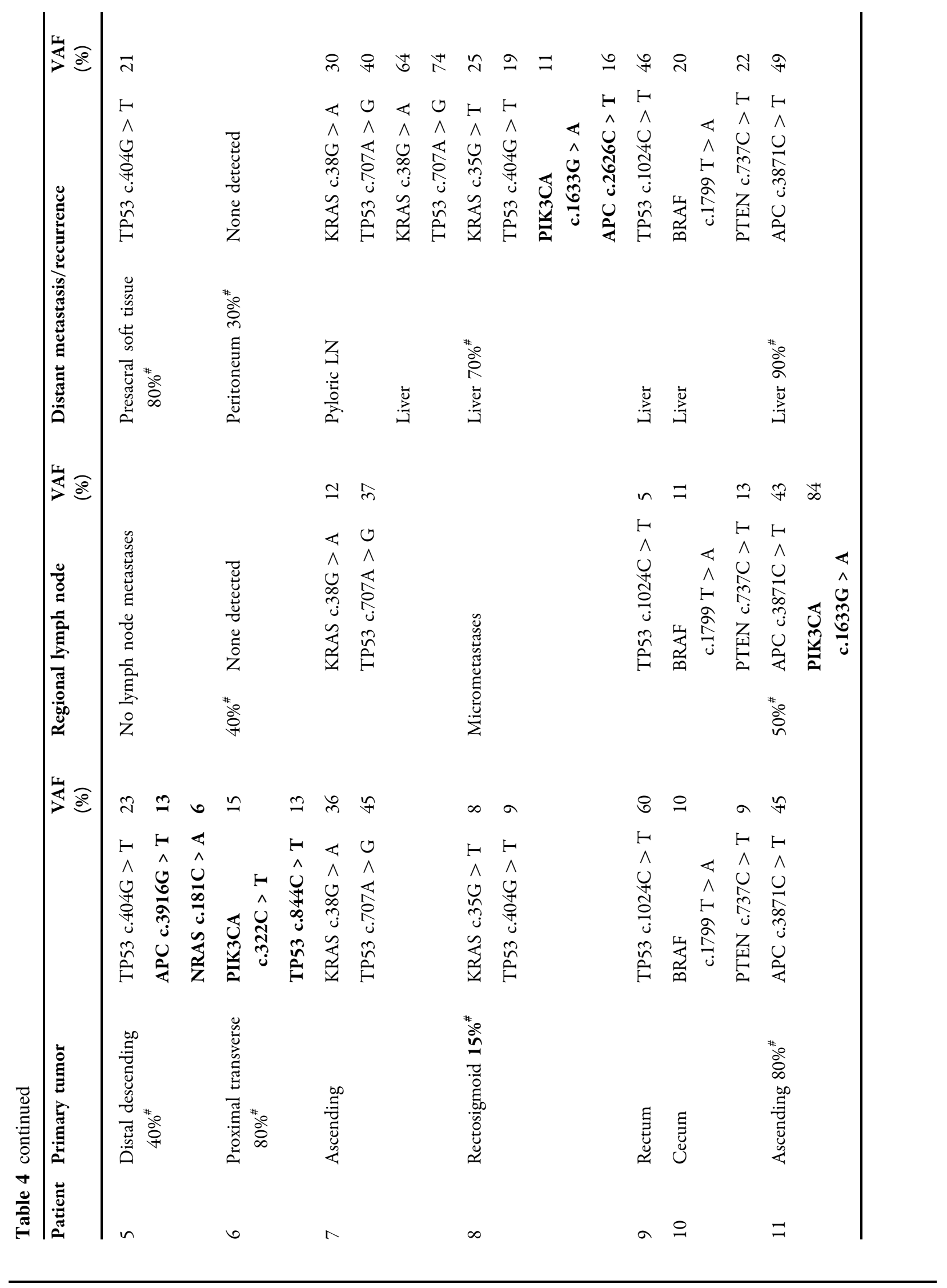




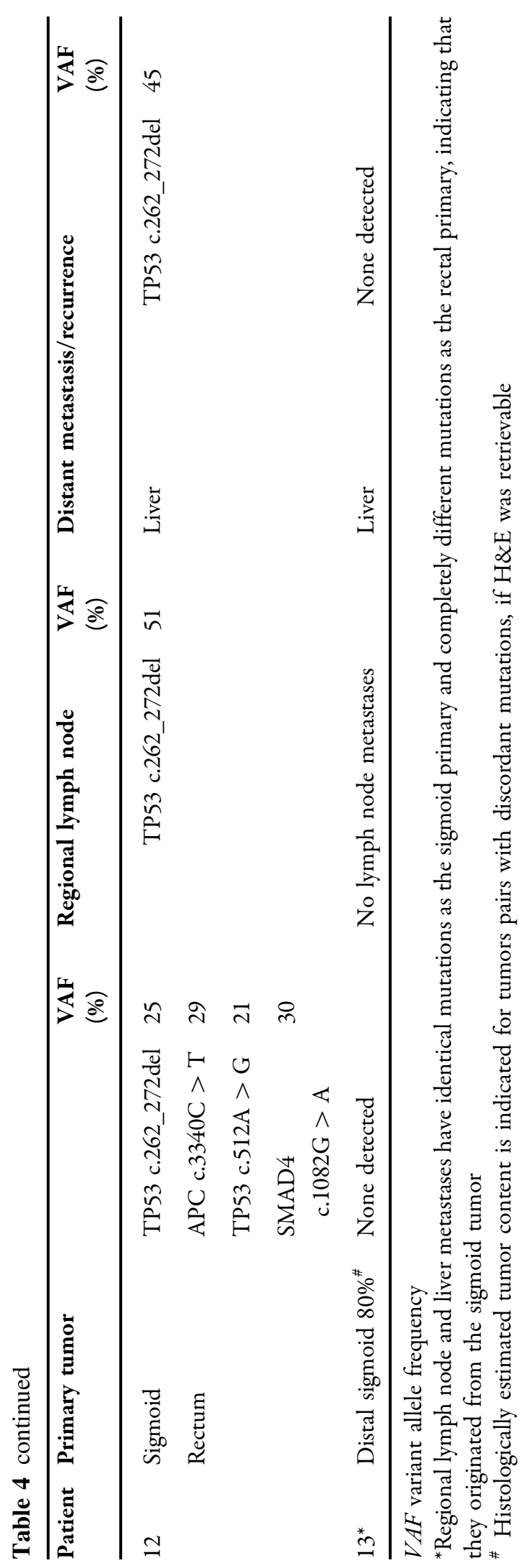

which were shared. Importantly, this pattern held true for PT-RLN pairs as well.

Since testing for activating mutations in $K R A S, N R A S$, and BRAF is recommended to predict response to FDA-approved therapies, we specifically evaluated concordance for mutations in this gene trio. For 12 of 13 (92\%) patients, their mutational status was concordant, and the choice of sample for biomarker testing would not have impacted eligibility for FDA-approved therapies (Tables 3, 4). KRAS and $B R A F$ mutations were detected in three and one patients, respectively, and were completely concordant. However, patient 5 had an NRAS mutation, which was detected only in the PT. Therefore, while there is generally a high degree of concordance between primary and metastatic tumor pairs for mutations in the gene trio, important actionable differences may occasionally exist.

Because NGS is also used to identify biomarkers for eligibility into clinical trials, we also examined discordances in other genes in the 50-gene panel (Table 3). Three of 12 (25\%) patients had at least one mutation detected in the PT but not in the DM (patients 5 and 6) or, conversely, in the RLN but not in the PT or DM (patient 11; Tables 3, 4). APC mutations which are considered early events in colorectal tumorigenesis were detected in four patients, and TP53 mutations which are considered late events were detected in eight patients. One patient was discordant for an $A P C$ mutation and another for a TP53 mutation. PIK3CA mutations were detected in three patients, and two had discordances.

One unique feature of our study is that we analyzed multiple metastatic sites, when available and suitable for sequencing, to address spatial intertumoral heterogeneity. Eleven of 13 patients had at least two metastatic and/or recurrent tumors sequenced, including patients 3,4 , and 7 who had three to five tumors. We found that mutational concordance was maintained spatially across multiple RLNs and DMs and temporally across synchronous and metachronous tumors (Table 4, Supplemental Table S1). 


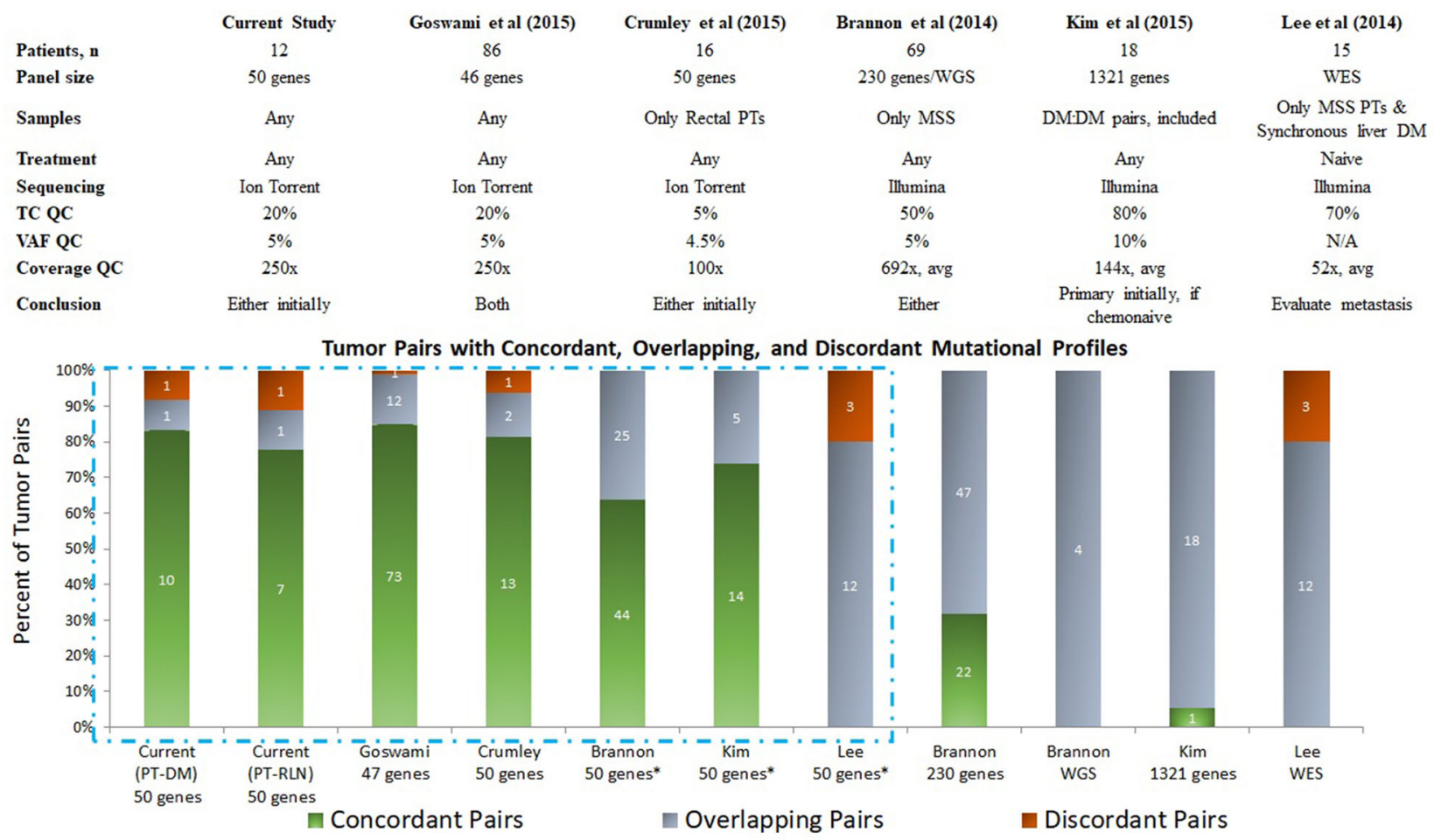

Fig. 3 Mutational concordance between tumor pairs across six studies. a Studies examining genetic differences in primary and metastatic CRCs were compared for variables that potentially explain differences in their results. Brannon et al. also performed WGS on four of their 69 patients. Kim et al. had one patient with two primarymetastatic pairs and two patients with only metastaticmetastatic pairs, all of which were included in the analysis

\section{Technical, Biological, and Clinical Factors Influence Mutational Discordance}

To explore potential causes of discordance, we first confirmed that each tumor in a pair was in fact derived from the same patient. All samples from the same patient but not samples from other patients in our cohort had an identical set of putative germline variants, thus excluding the possibility of a sample mix-up contributing to discordance (Supplement Figure S1). Secondly, all of our samples exceeded the laboratory-established minimum requirements to pass quality control, excluding poor sequencing quality as a significant cause of discordance. Even though the read depth cutoff was 250X, the average across samples was much higher at below. b Most studies find that patient-matched tumor pairs are highly concordant for mutations in common cancer genes. Numbers in bars represent the number of pairs in the respective category. $W G S$ whole genome sequencing, WES whole exome sequencing, ${ }^{*} \mathrm{Kim}$ et al. and Lee et al. studies reanalyzed after including only 50 genes interrogated in the current study. Compared studies are boxed in

2700X, and the sample with the lowest coverage had a mean of $1700 X$ coverage per amplicon. All of our reported variants also passed all quality metrics, and all were confirmed by Sanger sequencing or other method.

Next, we sought to determine whether sample inadequacy may have contributed to any of the discordances. Therefore, we revisited the $\mathrm{H} \& \mathrm{E}$ slides, when possible, from our discordant cases to re-evaluate the TC (Table 4). The liver metastasis in patient 4 had a TC of $10 \%$, which is below our laboratory's established QC limit of $20 \%$ TC, and likely accounts for the absence of ATM and TP53 mutations in liver metastasis. The possibility of two driver mutations being lost due to clonal evolution in the DM is highly 
unlikely, especially when three other metastases and both recurrences had the mutations.

In some instances, true biological factors may account for discordances. In patient 11 , for instance, the spatial intertumoral heterogeneity for the PIK3CA mutation may reflect branched evolution with the emergence of a novel variant in the RLN that is subsequently amplified. An APC mutation is present in the PT, RLN, and $\mathrm{DM}$ at a VAF of $\sim 45 \%$ in all three samples, suggesting it is a clonal heterozygous variant. The PIK3CA variant, however, was present at an even higher VAF of $84 \%$, suggesting that the mutant allele is amplified (loss of the wild-type allele is also explanatory but is less likely for oncogenes). The presence of a very minor subclone harboring the PIK3CA mutation in the PT that subsequently expanded in the RLN remains a formal possibility.

Intratumoral heterogeneity of the PT may also account for discordance between primaries and metastases. In patient 5 , the low VAF of the APC $(13 \%)$ and NRAS (6\%) mutations relative to the adequate TC (40\%) are suggestive of their presence in a small subclone within the PT. Regardless of whether this subclone arose before or after the metastatic event, it clearly did not contribute to the DM (80\% TC). A similar situation is found in patient 6 with low VAF for PIK3CA (15\%) and TP53 (13\%) relative to high TC $(80 \%)$ in the PT. However, these mutations were not detected in either the RLN (40\% TC) or DM (30\% TC), suggesting that these small clones did not contribute to the metastases.

The cause of the discordance may be multifactorial and may be elusive. In patient 8 , there are at least two possible causes for the absence of the PIK3CA and APC mutations in the PT. First, the low TC (15\%) of the PT suggests that these mutations may have been missed. Alternatively, spatial intertumoral heterogeneity may be explanatory. Since both mutations are subclonal (VAF of $11 \%$ for PIK3CA and $16 \%$ for $A P C)$ in the $\mathrm{DM}(70 \% \mathrm{TC})$, they may be emerging mutations entirely novel to the DM. Enrichment of tumor cells in the PT with laser capture micro-dissection would prove useful in teasing out these possibilities.

Finally, clinical factors are paramount when considering discordance between primaries and metastases. For patient 12, we initially only analyzed the rectal primary which is clearly genetically different from the RLN and DM. This apparent discordance was not resolved until careful review of the patient's records revealed a history of a second primary in the sigmoid colon. Sequencing the latter tumor revealed a TP53 mutation identical to the one found in the RLN and DM. In a clinical setting, excluding metastasis from a second primary, including a primary from another organ or cryptic primary, is critical.

\section{Meta-Analysis}

A total of five studies were included in the final meta-analysis. From each study, the total number of patients, the size of the NGS panel, sequencing parameters, and final conclusion were extracted (Fig. 3).

\section{DISCUSSION}

Pathologists and oncologists are often faced with difficult decisions of which sample to select and whether to collect a new sample for biomarker testing. These decisions can potentially influence downstream clinical management and patient outcomes. The NCCN guidelines state that either the PT or the DM may be tested for RAS and BRAF mutations due to the high concordance rate. Consistently, we find that $K R A S$ and $B R A F$ mutations were always concordant in our study. One patient, however, had discordant NRAS status, indicating that important potentially actionable differences may be discoverable by sequencing the other paired tumor.

The current NCCN guidelines make no specific recommendations for NGS, yet it has become common practice to comprehensively profile tumors using NGS. With a 50 cancer-related gene panel, we demonstrated that the majority of patient-matched primary and metastatic CRCs show a high degree of concordance. PT and metastatic tumors were concordant in $83 \%$, partially overlapping in $8 \%$, and entirely discordant in another $8 \%$ of patients. Our findings are consistent with those reported 
by Crumley et al. [26] and Goswami et al. [25] who used identical and similar NGS panels, respectively, and with Kim et al. [27] and Brannon et al. [28] after we re-evaluated their data by considering only the 50 genes on our panel (Fig. 3, Supplemental Figure S2). The proportion of patients whose tumor pairs had identical (64-85\%), overlapping (8-36\%), or completely discordant $(0-11 \%)$ mutations among the studies was variable, although tending towards concordance. Variability between these studies may be attributable to sample selection criteria, sequencing methodology (including gene panel size), and bioinformatic pipelines (Fig. 3). In contrast, Lee et al. [29] found a markedly high degree of discordance, even after we re-evaluated their data by considering only the 50 genes, compared to the above studies, and was a clear outlier for reasons that are unclear (Supplemental Figure S2). Based on all other studies, about 77\% [68-86\%] (95\% CI) of all tumor pairs are expected to have identical mutations when analyzed on a panel of about 50 common cancer genes (Supplemental Figure S3). In our study, we excluded from comparison samples with low TC, consistent with other studies, due to potential for false negatives. Expectedly, inclusion of these samples would have significantly increased the number of partially overlapping and entirely discordant PT-DM pairs from $8 \%$ each to $15 \%$ each. It confirms the importance of ensuring adequate sampling and TC in any sequenced sample to draw reliable results to genomically guide therapy. Based on our meta-analysis, we propose a set of sensible guidelines to aid pathologists and oncologists in specimen selection:

\section{Clinical Recommendations}

Situation 1: Only one sample is available and there is no plan for a second collection.

Recommendation: Attempt analyzing the available sample.

Considerations: While it is understood that any inadequate/low-quality samples carry the risk of false negatives, the cost and risk to the patient of resampling generally outweigh that of testing the available sample. Therefore, testing on the available sample should be attempted unless there is a strong reason to defer. If applicable, the final report should indicate that the sample was insufficient (low nucleic acid yield, noisy sequencing results, etc.) and suggest that a second collection be performed if clinically warranted.

\section{Situation 2: Multiple samples are available contemporaneously at the time sequencing is ordered, even if they were collected extemporaneously.}

Recommendation: Decide based on clinical indication and sample adequacy. Simultaneous sequencing of more than one sample is not generally recommended due to low yield and cost and resource considerations.

Considerations: Clinical factors should dominate decision making.

Initial therapy: Any sample may be considered for testing the primary resistance markers, KRAS, NRAS, and BRAF, because of high concordance. Therefore, specimen selection should be based on the possibility that the tumor may be negative for these markers. In this setting, specimen adequacy and/or clinical considerations should be the primary determinant for specimen selection. Whether metastasis is synchronous or metachronous, the time interval between the diagnosis of the primary and metastatic tumors, whether there was neoadjuvant therapy, whether the primary or metastasis was resected or simply biopsied, and whether the resection was complete or incomplete are potential considerations. However, the impact of these factors on sample selection remains to be elucidated on a case-by-case basis.

Acquired resistance: The resistant focus/tumor should be prioritized. If a sample is suboptimal due to factors related to sample processing (heavy-metal fixatives, harsh decalcifying agents, extended cold ischemic times, extended fixation times) or due to the sample itself (age of sample, cellularity, tumor purity), attempt testing and report if sample passed quality controls. 
Reflex testing of an alternate sample, however, may be considered if the initial analysis failed quality control, an actionable marker was not detected, or a resistant focus develops subsequently.

\section{Situation 3: Only one sample is available, but a second collection is planned before therapy initiation.}

Recommendation: Wait for pathology to review both samples.

Considerations: Unless immediate testing is clinically indicated, waiting on the availability of both samples for review is advised. Reflex to situation 2.

A small sample size reflects the most important limitation of our work. However, as we incorporate our data into a meta-analysis, we are able to provide a robust estimate of the genetic similarity of patient-matched tumors. Furthermore, our comprehensive analysis of every patient, including multiple metastases and recurrences, further substantiates our conclusion that primaries and metastases are generally genetically similar. While our study only focused on 50 genes, we do not consider this to be a limitation of significant importance given that the objective of our study was to determine differences in actionable gene mutations in primary and metastatic CRCs. At this time, the number of therapeutically actionable genes is small, and the vast majority are reflected in the 50-gene panel, and those that are not, such as NTRK, are exceptionally rare in CRC. While this study was conducted at a single institution, we do not believe this to be a major shortcoming to the generalizability of our study due to the diversity of our patient population.

Bridging our technical findings to the clinical arena, the advent of deep NGS profiling with large gene panels has illuminated previously unknown cancer complexity such as intratumoral heterogeneity, heterogeneity between primaries and metastases, tumor evolution over time and with treatment, and genetic heterogeneity across tumor types. The ultimate arbiter of significance of these testing activities is impact on care and outcomes. This study's use of a panel of genes frequently altered in solid tumors suggests that these discordances may be of clinical significance, especially when the RLN and/or DM sites display entirely discordant actionable mutations compared to their PT.

The clinical utility of KRAS, NRAS, and BRAF is certainly clear. In our meta-analysis, there were only rare RAS discordances and no $B R A F$ discordances. However, rare differences in the mutational status of these genes could have a significant impact on treatment. For instance, patient 5 had an NRAS mutation in the PT but not in the DM and, therefore, would have potentially qualified for anti-EGFR therapy. Among significantly altered CRC genes, TP53, $A P C, P I K 3 C A$, and SMAD4 were the most frequently discordant in our meta-analysis (Supplemental Table S2). Always a leader in frequency, mutated TP53 is a target of extremely high unmet need without well-established therapeutic options, but some inroads are in progress, including a phase 2 study of the antiviral agent lamivudine in patients with p53mutant mCRC (NCT03144804).

PIK3CA and the PI3K/AKT/MTOR pathway are also emerging therapeutic targets; there are already many FDA-approved indications for some tumor types and promising late-phase clinical trial therapies in advanced and/or refractory cancer patients. At the time of this writing, three trials evaluating drugs that target activating PIK3CA mutations in mCRC were in progress (NCT03006172, NCT02465060, NCT03711058). In our study, sequencing of the metastatic sites in patients 8 and 11 revealed a PIK3CA mutation not present in their PT. These PIK3CA mutations would have likely qualified them for enrollment, which would not have been possible if only the PT had been sequenced. Conversely, the lack of a detectable PIK3CA variant in either metastasis in patient 6 , although detected in the PT, would have potentially excluded this patient. Currently, there are no trials targeting APC or SMAD4. However, Zhang et al. [31] described a preclinical small molecule selective for killing cells carrying common truncating mutations of this gene (as are all APC variants shown in Table 4). Inactivation of APC results in the dysregulation of the Wnt signaling through beta-catenin, and several Wnt pathway 
inhibitors are in clinical trials for solid tumors [32]. In addition, beta-catenin activation confers synthetic lethality when tumor necrosis factor-related apoptosis-inducing ligand (TRAIL) is upregulated by agonists, which are also currently in clinical trials in some solid tumor types [33].

Finally, as it is an area of intense activity, we briefly discuss the utility of liquid biopsies in addressing the issue of sample selection. The analysis of liquid biopsies may provide a comprehensive view of the genomic landscape of multifocal disease [34]. Liquid biopsies include circulating tumor cells, exosomes, and circulating tumor DNA (ctDNA) and RNA. Of these, ctDNA has achieved the greatest advancement to the clinical arena [35]. The analysis of ctDNA offers several advantages over the analysis of tissue. One that is directly relevant to this manuscript pertains to its potential to capture intra- and intertumoral genetic heterogeneity. Because ctDNA reflects the released DNA from tumor cells throughout a given tumor mass (intratumoral heterogeneity) and across multiple tumor masses (spatial intertumoral heterogeneity) in a patient, its analysis in theory will reflect the total genetic composition of all tumor masses, which is in contrast to analysis of tissue $[36,37]$. While in our manuscript, we analyzed up to six tumor masses from a single patient, this is not feasible on a routine clinical basis. With ctDNA, such analyses of multiple sites may not be necessary. However, ctDNA has important drawbacks that has limited its use as a biospecimen, including higher failure rates due to insufficient quantity of ctDNA for analysis and limited analytical sensitivity. At this time, there are no FDA-approved ctDNA-based companion diagnostic tests for CRCs. In lung, breast, ovarian, and prostate cancer, however, FDA approval has been granted for select wellestablished biomarkers, but negative test results must be followed up with tissue-based tests due to the aforementioned limitations of ctDNAbased testing [35].

\section{CONCLUSIONS}

In summary, our own findings and our metaanalysis support that the initial analysis can be made on either the primary or metastatic tumor depending on availability, as there is a high degree of mutational concordance and because the occasional novel mutation may not always be actionable. Therefore, unless a biopsy of the mCRC is deemed necessary in the setting of resistance, the majority of patients will not need to be re-biopsied exclusively for identifying actionable novel mutations. We also showed that mutations are similar across various metastatic sites, including regional lymph nodes, and, therefore, the latter may be suitable alternative specimens for sequencing. However, we demonstrated that systematic evaluation of tissue and sequencing QC metrics, especially TC and $\mathrm{VAF}$, is paramount, as these are common causes of discordance. Therefore, if the initial analysis failed QC, additional samples, if and when available, should be considered for sequencing. For about a quarter of patients, additional mutations may be detected due to clonal evolution or tumor heterogeneity by sequencing additional samples, and for a smaller subset of these patients, findings may offer new opportunities to enroll in clinical trials.

\section{ACKNOWLEDGEMENTS}

Thanks to Drs. Rhona Schreck and Mark Ewalt for expert opinions and helpful discussions.

Funding. This work and the journal's rapid service fee were sponsored by the Department of Pathology and Laboratory Medicine at CedarsSinai Medical Center.

Authorship. All named authors meet the International Committee of Medical Journal Editors (ICMJE) criteria for authorship for this article, take responsibility for the integrity of the work as a whole, and have given their approval for this version to be published. 
Authors' Contributions. All authors provided review of the manuscript. S.B.P. and J.L. wrote the manuscript. S.B.P., R.B., N.F., M.C-D., and J.R.L. performed pathologic review of samples, analyzed genomic results, participated in experimental design, and provided various aspects of the manuscript text or figures. J.C.H. analyzed genomic results, helped with experimental design, and created tables. A.A., C.R., and A.P. performed wet bench analysis of samples. S.B.P., Z.L., and N.D. performed the statistical analysis.

Disclosures. The authors have no relevant disclosures. S.P. (HeloGenika LLC, Dexter, MI) changed affiliation during the study. The following authors changed affiliation after the study. R.B. (Adaptive Biotechnologies, Laboratory Operations, Seattle, WA, USA), N.F (Strateos, Menlo, CA, USA), M.C-D. (Université de Montréal, Département de pathologie et biologie cellulaire, Montreal, Quebec, Canada), A.P. (Kaiser Permanente, Department of Pathology, Los Angeles, CA, USA), C.R. (Tempus Labs, Inc., Laboratory Operations, Chicago, IL, USA), J.H. (Department of Medical and Molecular Genetics, Indiana University School of Medicine, Indianapolis, IN, USA), and Z.L. (Penn State Cancer Institute, Division of Biostatistics and Bioinformatics, Hershey, PA, USA).

Compliance with Ethics Guidelines. This work was approved by the Institutional Review Board at Cedars-Sinai Medical Center. The requirement for consent was waived.

Data Availability. The data sets generated during and/or analyzed during the current study are available from the corresponding author on reasonable request.

Open Access. This article is licensed under a Creative Commons Attribution-NonCommercial 4.0 International License, which permits any non-commercial use, sharing, adaptation, distribution and reproduction in any medium or format, as long as you give appropriate credit to the original author(s) and the source, provide a link to the Creative Commons licence, and indicate if changes were made. The images or other third party material in this article are included in the article's Creative Commons licence, unless indicated otherwise in a credit line to the material. If material is not included in the article's Creative Commons licence and your intended use is not permitted by statutory regulation or exceeds the permitted use, you will need to obtain permission directly from the copyright holder. To view a copy of this licence, visit http://creativecommons.org/licenses/by$\mathrm{nc} / 4.0 /$.

\section{REFERENCES}

1. Siegel RL, Miller KD, Fuchs HE, Jemal A. Cancer statistics, 2021. CA Cancer J Clin. 2021;71(1):7-33. https://doi.org/10.3322/caac.21654.

2. Siegel RL, Miller KD, Goding Sauer A, et al. Colorectal cancer statistics, 2020. CA Cancer J Clin. 2020;70(3):145-64. https://doi.org/10.3322/caac. 21601.

3. National Comprehensive Cancer Network. Clinical practice guidelines in oncology: Colon Cancer Version 2.2020. https://www.nccn.org/ professionals/physician_gls/pdf/colon.pdf.

4. National Comprehensive Cancer Network. Clinical practice guidelines in oncology: Rectal Cancer Version 2.2020. https://www.nccn.org/ professionals/physician_gls/pdf/rectal.pdf.

5. Marques AM, Turner A, de Mello RA. Personalizing medicine for metastatic colorectal cancer: current developments. World J Gastroenterol. 2014;20(30): 10425-31. https://doi.org/10.3748/wjg.v20.i30. 10425.

6. Hayashi Y, Widjono YW, Ohta K, et al. Expression of EGF, EGF-receptor, p53, v-erb B and ras p21 in colorectal neoplasms by immunostaining paraffinembedded tissues. Pathol Int. 1994;44(2):124-30.

7. Koretz K, Schlag P, Moller P. Expression of epidermal growth factor receptor in normal colorectal mucosa, adenoma, and carcinoma. Virchows Arch Pathol Anat Histopathol. 1990;416(4):343-9.

8. Sorich MJ, Wiese MD, Rowland A, Kichenadasse G, McKinnon RA, Karapetis CS. Extended RAS mutations and anti-EGFR monoclonal antibody survival benefit in metastatic colorectal cancer: a metaanalysis of randomized, controlled trials. Ann Oncol. 2015;26(1):13-21. https://doi.org/10.1093/ annonc/mdu378. 
9. Van Cutsem E, Huijberts S, Grothey A, et al. Binimetinib, encorafenib, and cetuximab triplet therapy for patients with BRAF V600E-mutant metastatic colorectal cancer: safety lead-in results from the phase III BEACON colorectal cancer study. J Clin Oncol. 2019;37(17):1460-9. https://doi.org/ 10.1200/jco.18.02459.

10. Sepulveda AR, Hamilton SR, Allegra CJ, et al. Molecular biomarkers for the evaluation of colorectal cancer: guideline from the American society for clinical pathology, college of American Pathologists, association for molecular pathology, and American society of clinical oncology. J Mol Diagn. 2017;19(2):187-225. https://doi.org/10.1016/j. jmoldx.2016.11.001.

11. Bhullar DS, Barriuso J, Mullamitha S, Saunders MP, O'Dwyer ST, Aziz O. Biomarker concordance between primary colorectal cancer and its metastases. EBioMedicine. 2019;40:363-74. https://doi. org/10.1016/j.ebiom.2019.01.050.

12. Siyar Ekinci A, Demirci U, Cakmak Oksuzoglu B, et al. KRAS discordance between primary and metastatic tumor in patients with metastatic colorectal carcinoma. J BUON. 2015;20(1):128-35.

13. Watanabe $\mathrm{T}$, Kobunai $\mathrm{T}$, Yamamoto $\mathrm{Y}$, et al. Heterogeneity of KRAS status may explain the subset of discordant KRAS status between primary and metastatic colorectal cancer. Dis Colon Rectum. 2011;9:1170-8.

14. Kim MJ, Lee HS, Kim JH, et al. Different metastatic pattern according to the KRAS mutational status and site-specific discordance of KRAS status in patients with colorectal cancer. BMC Cancer. 2012;12:347. https://doi.org/10.1186/1471-240712-347.

15. Gattenlohner S, Etschmann B, Kunzmann V, et al. Concordance of KRAS/BRAF mutation status in metastatic colorectal cancer before and after AntiEGFR therapy. J Oncol. 2009;2:831626. https://doi. org/10.1155/2009/831626.

16. Jin Z, Sinicrope FA. Prognostic and predictive values of mismatch repair deficiency in non-metastatic colorectal cancer. Cancers (Basel). 2021;13(2):4. https://doi.org/10.3390/cancers13020300.

17. Franke AJ, Skelton WP, Starr JS, et al. Immunotherapy for colorectal cancer: a review of current and novel therapeutic approaches. J Natl Cancer Inst. 2019;111(11):1131-41. https://doi.org/ 10.1093/jnci/djz093.

18. Jung J, Kang Y, Lee YJ, et al. Comparison of the mismatch repair system between primary and metastatic colorectal cancers using immunohistochemistry. J Pathol Transl Med. 2017;51(2):129-36. https://doi.org/10.4132/jptm. 2016.12.09.

19. Wang Z, Tang X, Wu X, Yang M, Wang D. Mismatch repair status between primary colorectal tumor and metastatic tumor, a retrospective consistent study. Biosci Rep. 2019;39(12):120-34. https://doi.org/10.1042/bsr20190730.

20. Fujiyoshi K, Yamamoto G, Takahashi A, et al. High concordance rate of KRAS/BRAF mutations and MSI-H between primary colorectal cancer and corresponding metastases. Oncol Rep. 2017;37(2): 785-92. https://doi.org/10.3892/or.2016.5323.

21. Haraldsdottir S, Roth R, Pearlman R, Hampel H, Arnold CA, Frankel WL. Mismatch repair deficiency concordance between primary colorectal cancer and corresponding metastasis. Fam Cancer. 2016;15(2):253-60. https://doi.org/10.1007/ s10689-015-9856-2.

22. He WZ, Hu WM, Wang F, et al. Comparison of mismatch repair status between primary and matched metastatic sites in patients with colorectal cancer. J Natl Compr Canc Netw. 2019;17(10): 1174-83. https://doi.org/10.6004/jnccn.2019.7308.

23. Damodaran S, Berger MF, Roychowdhury S. Clinical tumor sequencing: opportunities and challenges for precision cancer medicine. Am Soc Clin Oncol Educ Book. 2015;6:175-82.

24. Fang W, Radovich M, Zheng Y, et al. 'Druggable' alterations detected by Ion Torrent in metastatic colorectal cancer patients. Oncol Lett. 2014;6: 1761-6.

25. Goswami RS, Patel KP, Singh RR, et al. Hotspot mutation panel testing reveals clonal evolution in a study of 265 paired primary and metastatic tumors. Clin Cancer Res. 2015;21(11):2644-51. https://doi. org/10.1158/1078-0432.ccr-14-2391.

26. Crumley SM, Pepper KL, Phan AT, Olsen RJ, Schwartz MR, Portier BP. Next-generation sequencing of matched primary and metastatic rectal adenocarcinomas demonstrates minimal mutation gain and concordance to colonic adenocarcinomas. Arch Pathol Lab Med. 2015. https:// doi.org/10.5858/arpa.2015-0261-SA.

27. Kim R, Schell MJ, Teer JK, Greenawalt DM, Yang M, Yeatman TJ. Co-evolution of somatic variation in primary and metastatic colorectal cancer may expand biopsy indications in the molecular era. PLOS ONE. 2015;10(5):e0126670. https://doi.org/ 10.1371/journal.pone.0126670.

28. Brannon AR, Vakiani E, Sylvester BE, et al. Comparative sequencing analysis reveals high genomic 
concordance between matched primary and metastatic colorectal cancer lesions. Genome Biol. 2014;15(8):454. https://doi.org/10.1186/s13059014-0454-7.

29. Lee SY, Haq F, Kim D, et al. Comparative genomic analysis of primary and synchronous metastatic colorectal cancers. PLoS ONE. 2014;3:e90459.

30. Brosens RP, Belt EJ, Haan JC, Buffart TE, Carvalho B, Grabsch H, Quirke P, Cuesta MA, Engel AF, Ylstra B, Meijer GA. Comprehensive molecular characterization of human colon and rectal cancer. Nature. 2012;487(7407):330-7. https://doi.org/10.1038/ nature11252.

31. Zhang L, Theodoropoulos PC, Eskiocak U, et al. Selective targeting of mutant adenomatous polyposis coli (APC) in colorectal cancer. Sci Transl Med. 2016;8(361):361ra140. https://doi.org/10. 1126/scitranslmed.aaf8127.

32. Lu W, Tinsley HN, Keeton A, Qu Z, Piazza GA, Li Y. Suppression of Wnt/beta-catenin signaling inhibits prostate cancer cell proliferation. Eur J Pharmacol. 2009;602(1):8-14. https://doi.org/10.1016/j.ejphar. 2008.10.053.
33. Zhang L, Ren X, Alt E, et al. Chemoprevention of colorectal cancer by targeting APC-deficient cells for apoptosis. Nature. 2010;464(7291):1058-61. https://doi.org/10.1038/nature08871.

34. Heidrich I, Ačkar L, Mossahebi Mohammadi P, Pantel K. Liquid biopsies: potential and challenges. Int J Cancer. 2021;148(3):528-45. https://doi.org/ 10.1002/ijc.33217.

35. Goodsaid FM. The labyrinth of product development and regulatory approvals in liquid biopsy diagnostics. Clin Transl Sci. 2019;12(5):431-9. https://doi.org/10.1111/cts.12657.

36. Huang HM, Li HX. Tumor heterogeneity and the potential role of liquid biopsy in bladder cancer. Cancer Commun (Lond). 2021;41(2):91-108. https://doi.org/10.1002/cac2.12129.

37. Russano M, Napolitano A, Ribelli G, et al. Liquid biopsy and tumor heterogeneity in metastatic solid tumors: the potentiality of blood samples. J Exp Clin Cancer Res. 2020;39(1):95. https://doi.org/10. 1186/s13046-020-01601-2. 